MITSUBISHI ELECTRIC RESEARCH LABORATORIES

http://www.merl.com

\title{
Unified Analysis of Linear Block Precoding for Distributed Antenna Systems
}

\author{
Toshiaki Koike-Akino, Andreas Molisch, Zhifeng Tao, Philip Orlik, Toshiyuki Kuze
}

TR2009-077 December 2009

\begin{abstract}
Block transmission with cyclic prefix is a promising technique to realize high-speed data rates in frequency selective fading channels. Many popular linear precoding schemes, including orthogonal frequency-division multiplexing (OFDM), single-carrier (SC) block transmission, and time-reversal (TR), can be interpreted as such a block transmission. This paper presents a unified performance analysis which shows how the optimal precoding strategy depends on the receiver type and the optimization criterion (capacity and mean-square error). We analyze three variants of TR methods (based on maximum-ratio combining, equal-gain combining and selective combining) and two-types of pre-equalization methods (zero-forcing and minimum mean-square error). As one application of our framework, we derive optimal power control for OFDM in the presence of interference limitation for distributed antenna systems; we find that without power control, OFDM does not have any capacity advantage over SC transmissions. When comparing SC and TR, we find that for single-antenna systems at high SNRs, SC has a capacity advantage; however, TR performs better in the low SNR regime. For multiple-antenna systems, TR always provides higher capacity, and the capacity of TR can approach that of optimal precoders with a number of distributed antennas.
\end{abstract}

Globecom 2009

\footnotetext{
This work may not be copied or reproduced in whole or in part for any commercial purpose. Permission to copy in whole or in part without payment of fee is granted for nonprofit educational and research purposes provided that all such whole or partial copies include the following: a notice that such copying is by permission of Mitsubishi Electric Research Laboratories, Inc.; an acknowledgment of the authors and individual contributions to the work; and all applicable portions of the copyright notice. Copying, reproduction, or republishing for any other purpose shall require a license with payment of fee to Mitsubishi Electric Research Laboratories, Inc. All rights reserved.
}

Copyright (C) Mitsubishi Electric Research Laboratories, Inc., 2009

201 Broadway, Cambridge, Massachusetts 02139 



\title{
Unified Analysis of Linear Block Precoding for Distributed Antenna Systems
}

\author{
Toshiaki Koike-Akino*, Andreas F. Molisch ${ }^{\dagger}$, Zhifeng Tao ${ }^{\ddagger}$, Philip Orlik ${ }^{\ddagger}$, and Toshiyuki Kuze ${ }^{\S}$ \\ * JSPS Research Fellow, SEAS, Harvard University, 33 Oxford Street, Cambridge, MA 02138, U.S.A. \\ $\dagger$ Depart. of Electrical Engineering, University of Southern California, 3740 McClintock Av., Los Angeles, CA 90089, U.S.A. \\ $\ddagger$ Mitsubishi Electric Research Labs (MERL), 201 Broadway, Cambridge, MA 02139, U.S.A. \\ $\S$ Mitsubishi Electric Corporation, 5-1-1 Ofuna Kamakura, Kanagawa 247-8501, Japan \\ Email: koike@seas.harvard.edu, molisch@usc.edu, \{tao, porlik\}@merl.com
}

\begin{abstract}
Block transmission with cyclic prefix is a promising technique to realize high-speed data rates in frequency selective fading channels. Many popular linear precoding schemes, including orthogonal frequency-division multiplexing (OFDM), single-carrier (SC) block transmission, and time-reversal (TR), can be interpreted as such a block transmission. This paper presents a unified performance analysis which shows how the optimal precoding strategy depends on the receiver type and the optimization criterion (capacity and mean-square error). We analyze three variants of TR methods (based on maximum-ratio combining, equal-gain combining and selective combining) and two-types of pre-equalization methods (zero-forcing and minimum mean-square error). As one application of our framework, we derive optimal power control for OFDM in the presence of interference limitation for distributed antenna systems; we find that without power control, OFDM does not have any capacity advantage over SC transmissions. When comparing SC and TR, we find that for single-antenna systems at high SNRs, SC has a capacity advantage; however, TR performs better in the low SNR regime. For multiple-antenna systems, TR always provides higher capacity, and the capacity of TR can approach that of optimal precoders with a number of distributed antennas.
\end{abstract}

\section{INTRODUCTION}

As data rates are constantly increasing, wireless transmission systems require larger bandwidths, which make them more susceptible to the effects of the frequency selectivity of the propagation channels [1]. Several transmission schemes are used for fourth-generation cellular and other advanced wireless communications systems to cope with this situation:

- Multi-carrier transmission, in particular orthogonal frequency-division multiplexing (OFDM) has been adopted for most high data-rate wireless standards, including 3GPP LTE, Wimax, and WiFi (802.11a, g, n).

- Single-carrier (SC) block transmission, combined with frequency-domain equalizations, is used in situations where the transmitter needs to avoid high peak-toaverage-power ratios, e.g., a cellular (3GPP LTE) handset.

- Time-reversal (TR) is another transmission technique that has gathered great interest in the past years [2-15] since it has a potential to decrease effective channel length and to enable the construction of simplified receivers.

It can be seen that all of the above techniques can be interpreted as linear block precoding schemes using cyclic prefix
(CP); the choice of precoder determines whether OFDM, SC, or TR (and which specific version of those methods) is used. While there is extensive literature dealing with each of those methods separately, there are - to the authors' best knowledge - no unified performance analyses, no comparisons amongst them, and no investigations of theoretical performance measures like link capacity.

In this paper, we present a unified performance analysis for various types of linear block precoding schemes, including several TR schemes, OFDM, SC transmissions (or, unitary precoding), and pre-equalization schemes. For each of those schemes, we optimize the precoder for i) maximizing capacity, and ii) minimizing mean-square error (MSE) for minimum MSE (MMSE) receivers. Multiple distributed transmitting antennas, as well as limits on the interference power to other ("victim") receivers, are taken into account. We provide several examples for the usefulness of our framework:

- We derive an optimal precoding scheme, which requires modified water-filling for power allocation, in distributedantenna multi-user systems.

- We show that at low SNRs, TR always performs better than SC (or, unitary precoding), but that in the singleantenna case SC provides higher capacity than TR at high SNRs if the cyclic prefix is long enough.

- It is confirmed that TR performs better than other schemes in terms of capacity and MSE performances when we exploit multiple distributed antennas for transmissions.

- We derive a scaling law which shows how the use of multiple antennas can increase the capacity of TR compared with that of SC.

The remainder of the paper is organized as follows: Section II sets up the system model and the mathematical formulation of precoders. Section III derives the optimum precoder among all the possible linear block precoders; subsequently Section IV derives a capacity scaling law which presents the impact of multiple antennas on the capacity advantage of TR over SC. In Section V, capacity and MSE comparisons are followed by a summary and conclusions. 


\section{LINEAR BLOCK PRECODING}

\section{A. Distributed Antenna Systems}

Fig. 1 shows the system model, in which we consider $M$ distributed transmitters and one intended receiver as well as $N-1$ possible victim receivers, all of which are equipped with only one antenna. We focus on block transmissions, where one transmitting block consists of $L$ information symbols and an $L_{\mathrm{cp}}$-symbol CP. We assume the channel remains constant over the duration of a block. The precoding is done based on the instantaneous channel impulse responses. We assume a simplified (tap-spaced) channel model with independent Rayleigh fading of each tap: Let $h_{n, m}(p) \in \mathbb{C}$ be the channel impulse response for $p T_{\mathrm{s}}$ delayed wave $\left(0 \leq p<P_{\mathrm{ch}}\right)$ from the $m$-th transmitter to the $n$-th receiver for $1 \leq m \leq M$ and $1 \leq n \leq N$. Here, $T_{\mathrm{s}}$ is the symbol duration.

Through a backbone network, source data is distributed to all the $M$ transmitters. At each transmitter, the corresponding modulation data $\boldsymbol{x} \in \mathbb{C}^{L \times 1}$ is multiplied with a block linear precoding matrix $\boldsymbol{P}_{m} \in \mathbb{C}^{L \times L}$. After padding with the $\mathrm{CP}$, the precoded block is transmitted to the $n$-th receiver, which in turn discards the $\mathrm{CP}$ and obtains

$$
\boldsymbol{y}_{n}=\sum_{m=1}^{M} \boldsymbol{H}_{n, m} \boldsymbol{P}_{m} \boldsymbol{x}+\boldsymbol{z}_{n} \triangleq \boldsymbol{H}_{n} \boldsymbol{x}+\boldsymbol{z}_{n},
$$

where $\boldsymbol{y}_{n} \in \mathbb{C}^{L \times 1}, \boldsymbol{H}_{n, m} \in \mathbb{C}^{L \times L}$, and $\boldsymbol{z}_{n} \in \mathbb{C}^{L \times 1}$ denote the received signal sequence, the (time domain) channel matrix, and the additive white Gaussian noise, respectively. We assume $\mathbb{E}\left[\boldsymbol{z}_{n} \boldsymbol{z}_{n}^{\dagger}\right]=\sigma^{2} \boldsymbol{I}_{L}, \mathbb{E}\left[\boldsymbol{x} \boldsymbol{x}^{\dagger}\right]=\boldsymbol{I}_{L}$. For the simulation in Section $\mathrm{V}$, we make further assumptions about the channel statistics; namely that each delay tap is Rayleigh fading with an exponential decay, and $\mathbb{E}\left[\boldsymbol{H}_{n, m}^{\dagger} \boldsymbol{H}_{n, m}\right]=\boldsymbol{I}_{L}$ (which means that the path loss is identical for all the channel links).

With a sufficiently long CP, we can write the channel matrix in the cyclic Toeplitz matrix form:

$$
\boldsymbol{H}_{n, m}=\sum_{p=0}^{P_{\mathrm{ch}}-1} h_{n, m}(p) \boldsymbol{\Pi}^{p},
$$

where $\Pi \in \mathbb{Z}_{2}^{L \times L}$ being a cyclic shift matrix, defined as

$$
\boldsymbol{\Pi}=\left[\begin{array}{ccccc}
0 & 0 & \cdots & 0 & 1 \\
1 & 0 & \ddots & \ddots & 0 \\
0 & 1 & \ddots & \ddots & \vdots \\
\vdots & \ddots & \ddots & \ddots & 0 \\
0 & \cdots & 0 & 1 & 0
\end{array}\right] .
$$

The channel matrix $\boldsymbol{H}_{n, m}$ is related to the (diagonal) frequency-domain channel matrix $\boldsymbol{G}_{n, m} \in \mathbb{C}^{L \times L}$ as

$$
\boldsymbol{H}_{n, m}=\boldsymbol{F G}_{n, m} \boldsymbol{F}^{\dagger},
$$

where the unitary matrix $\boldsymbol{F} \in \mathbb{C}^{L \times L}$ denotes the discrete Fourier transform, whose $(k, l)$-th entry is expressed as $\frac{1}{\sqrt{L}} \exp (-\jmath 2 \pi k l / L)$.

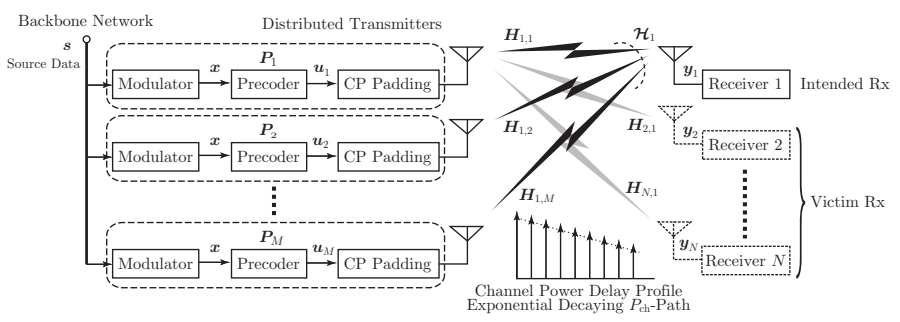

Fig. 1. Distributed antenna system with linear block precoding.

\section{B. Precoding Matrix}

Through appropriate choice of the precoder matrix $\boldsymbol{P}_{m}$, the above signal model can represent many types of block transmission schemes including SC, OFDM, TR, etc.

1) Single-Carrier (SC) Block Transmission: The precoding matrix for the SC block transmission is given as

$$
\boldsymbol{P}_{m}=\eta_{m} \boldsymbol{I}_{L},
$$

where $\eta_{m}$ corresponds to the transmission energy per symbol. The SC transmission is one of unitary precoding schemes.

2) Multi-Carrier OFDM Signalling: The precoding matrix for OFDM signalling is expressed as

$$
\boldsymbol{P}_{m}=\boldsymbol{F} \boldsymbol{\Phi}_{m}
$$

where a diagonal matrix $\boldsymbol{\Phi}_{m}$ denotes per-subcarrier power allocation at the $m$-th transmitter. Optimal power allocation is generally derived by water filling. If we cannot adopt such a power control (no channel state information at the transmitter), we should use a constant power allocation: $\boldsymbol{\Phi}_{m}=\eta_{m} \boldsymbol{I}_{L}$. In this case, it becomes one of unitary precoding schemes.

3) Time-Reversal (TR) Precoding: The original TR scheme has the following precoding matrix:

$$
\boldsymbol{P}_{m}=\eta_{m} \boldsymbol{H}_{1, m}^{\dagger} \text {. }
$$

Because it is similar to the pre-equalization based on maximum-ratio combining (MRC), we refer to it as MRC-TR.

In [7], a one-bit TR scheme is introduced, in which the precoding only controls the phase of the channel response. Its precoding matrix is expressed as follows:

$$
\boldsymbol{P}_{m}=\eta_{m}\left(\sum_{p=0}^{P_{\mathrm{ch}}-1} \frac{h_{1, m}(p)}{\left|h_{1, m}(p)\right|} \boldsymbol{\Pi}^{p}\right)^{\dagger} \triangleq \eta_{m} \breve{\boldsymbol{H}}_{1, m}^{\dagger},
$$

which only requires phase information of the channel impulse response. Since this TR scheme performs in the same way of the pre-equalization based on equal-gain combining (EGC), we call it EGC-TR hereafter.

Similarly, we can define a TR scheme based on selective $B$-branch combining (SLC-TR) as follows:

$$
\boldsymbol{P}_{m}=\eta_{m}\left(\sum_{b=0}^{B-1} h_{1, m}\left(p_{b}\right) \boldsymbol{\Pi}^{p_{b}}\right)^{\dagger},
$$

where $p_{b}$ represents the path index whose channel strength $\left|h_{1, m}\left(p_{b}\right)\right|^{2}$ is the $b$-th largest one. It is a kind of the pre-rake method. In this paper, we set $B=2$ for simplicity. 
4) Linear Pre-equalization: We consider pre-equalization schemes based on zero forcing (ZF) and MMSE criteria, whose precoder matrices are respectively expressed as follows:

$$
\begin{aligned}
& \boldsymbol{P}_{m}=\eta_{m} \boldsymbol{H}_{1, m}^{\dagger}\left(\boldsymbol{H}_{1, m} \boldsymbol{H}_{1, m}^{\dagger}\right)^{-1}, \\
& \boldsymbol{P}_{m}=\eta_{m} \boldsymbol{H}_{1, m}^{\dagger}\left(\boldsymbol{H}_{1, m} \boldsymbol{H}_{1, m}^{\dagger}+\sigma^{2} \boldsymbol{I}_{L}\right)^{-1} .
\end{aligned}
$$

\section{Performance Measures: Capacity and MSE}

This paper considers two types of receiver algorithms: an optimal equalizer and an MMSE linear equalizer. We focus on the achievable link capacity for optimal receivers and the MSE performance for MMSE receivers.

1) Capacity for Optimal Equalization: If the transmitter optimally controls the transmission rate for an optimal equalizer, the achievable capacity is bounded by

$$
C=\frac{1}{L} \log \operatorname{det}\left(\boldsymbol{I}_{L}+\frac{1}{\sigma^{2}} \mathcal{H}_{1} \mathcal{H}_{1}^{\dagger}\right),
$$

which is normalized by the bandwidth of $1 / T_{\mathrm{s}}$. Here, we neglect the loss of spectral efficiency due to the CP. Note that the capacity equation can employ either time- or frequencydomain representations of the channel, since $\operatorname{det}\left(\boldsymbol{F} \boldsymbol{A} \boldsymbol{F}^{\dagger}\right)=$ $\operatorname{det}(\boldsymbol{A})$. The Ergodic capacity is obtained by averaging the capacity above for all the possible channel realizations.

2) MSE for MMSE Equalization: The MMSE linear equalization is known as a low-complexity algorithm especially in the frequency-domain operation. The MMSE equalization achieves the following MSE:

$$
\varepsilon=\frac{1}{L} \operatorname{tr}\left[\left(\boldsymbol{I}_{L}+\frac{1}{\sigma^{2}} \mathcal{H}_{1}^{\dagger} \mathcal{H}_{1}\right)^{-1}\right] .
$$

\section{Optimal Precoder}

In this section, we derive an optimal precoding which is based on two different criteria; to maximize capacity and to minimize MSE. We can see that these optimal precoders are both OFDM signals. However, the optimal power allocation differs from each other.

\section{A. Optimal Precoder for Maximizing Capacity}

We first derive an optimal precoder which maximizes capacity constrained on the transmission power and the interference to victim receivers. The optimization problem is described as

$$
\begin{array}{ll}
\max _{\left\{\boldsymbol{P}_{m}\right\}} & \frac{1}{L} \log \operatorname{det}\left(\boldsymbol{I}_{L}+\frac{1}{\sigma^{2}} \boldsymbol{\mathcal { H }}_{1} \boldsymbol{\mathcal { H }}_{1}^{\dagger}\right), \\
\text { s.t. } & \frac{1}{L M} \sum_{m=1}^{M} \operatorname{tr}\left[\boldsymbol{P}_{m} \boldsymbol{P}_{m}^{\dagger}\right] \leq E_{\mathrm{s}}, \\
& \frac{1}{L M} \operatorname{tr}\left[\mathcal{H}_{n} \boldsymbol{H}_{n}^{\dagger}\right] \leq E_{\mathrm{v}}, \quad \text { for all } 2 \leq n \leq N,
\end{array}
$$

with $E_{\mathrm{s}}$ being the maximum symbol energy for transmission and $E_{\mathrm{v}}$ being the allowable interference limit at each victim receivers. Using the Lagrange multipliers method, we obtain the KKT condition for the saddle point as

$$
\boldsymbol{H}_{1, m}^{\dagger} \mathcal{H}_{1}\left(\sigma^{2} \boldsymbol{I}_{L}+\mathcal{H}_{1}^{\dagger} \boldsymbol{H}_{1}\right)^{-1}=\lambda_{1} \boldsymbol{P}_{m}+\sum_{n=2}^{N} \lambda_{n} \boldsymbol{H}_{n, m}^{\dagger} \boldsymbol{H}_{n},
$$

where $\lambda_{n}$ are the Lagrangian multipliers. It can be found that the solution is written in the form of $\boldsymbol{P}_{m}=\boldsymbol{F} \boldsymbol{\Phi}_{m}$. It indicates that the optimal precoding is OFDM with appropriate persubcarrier power control even for distributed antenna systems.

Let $G_{n, m}(i)$ and $\Phi_{m}(i)$ be the $i$-th diagonal element of the frequency-domain channel matrix $\boldsymbol{G}_{m, n}$ and the power allocation matrix $\boldsymbol{\Phi}_{m}$, respectively. When we define

$$
\Psi_{n}(i)=\sum_{m=1}^{M} G_{n, m}(i) \Phi_{m}(i)
$$

we can obtain the solution of optimal power control as

$$
\phi(i)=\frac{\Psi_{1}(i)}{\sigma^{2}+\left|\Psi_{1}(i)\right|^{2}} \underbrace{\left(\lambda_{1} \boldsymbol{I}_{M}+\boldsymbol{G}^{\dagger}(i) \boldsymbol{\Lambda} \boldsymbol{G}(i)\right)^{-}}_{\triangleq \boldsymbol{\Theta}(i)} \boldsymbol{g}(i),
$$

where $[\cdot]^{-}$denotes the pseudo inverse, and

$$
\begin{aligned}
\boldsymbol{\phi}(i) & =\left[\begin{array}{c}
\Phi_{1}(i) \\
\vdots \\
\Phi_{M}(i)
\end{array}\right], \quad \boldsymbol{g}(i)=\left[\begin{array}{c}
G_{1,1}^{*}(i) \\
\vdots \\
G_{1, M}^{*}(i)
\end{array}\right], \\
\boldsymbol{G}(i) & =\left[\begin{array}{cccc}
G_{1,1}(i) & G_{1,2}(i) & \cdots & G_{1, M}(i) \\
\vdots & \vdots & \cdots & \vdots \\
G_{N, 1}(i) & G_{N, 2}(i) & \cdots & G_{N, M}(i)
\end{array}\right], \\
\boldsymbol{\Lambda} & =\operatorname{diag}\left(0, \lambda_{2}, \ldots, \lambda_{N}\right) .
\end{aligned}
$$

Since $\Psi_{1}(i)=\boldsymbol{g}^{\dagger}(i) \boldsymbol{\phi}(i)$, the optimal $\Psi_{1}(i)$ must fulfil

$$
\left|\Psi_{1}(i)\right|^{2}=\left(\xi(i)-\sigma^{2}\right)_{+0},
$$

where $(x)_{+a}=\max (x, a)$ and $\xi(i)=\boldsymbol{g}^{\dagger}(i) \boldsymbol{\Theta}(i) \boldsymbol{g}(i)$. The Lagrangian multipliers $\lambda_{n}$ are chosen for satisfying (15) and (16). Note that this optimal power control is not given by the conventional water filling unless $\lambda_{n}=0$ for all $2 \leq n \leq N$. Without interference limitation, the $m$-th entry of the optimal power allocation in (18) reduces to

$$
\Phi_{m}(i)=\sqrt{\left(\frac{1}{\lambda_{1}}-\frac{\sigma^{2}}{\|\boldsymbol{g}(i)\|^{2}}\right)_{+0}} \frac{G_{1, m}^{*}(i)}{\|\boldsymbol{g}(i)\|},
$$

which means that distributed antennas should form the MRC diversity transmissions and use the conventional water filling for power allocation. With the optimal precoder, the maximized capacity is expressed as

$C=\frac{1}{L} \sum_{i=0}^{L-1} \log \left(1+\frac{1}{\sigma^{2}}\left|\Psi_{1}(i)\right|^{2}\right)=\frac{1}{L} \sum_{i=0}^{L-1}\left(\log \left(\frac{\xi(i)}{\sigma^{2}}\right)\right)_{+0}$.

\section{B. Optimal Precoder for Minimizing MSE}

In a similar way, an optimal precoder which minimizes the MSE, when the receiver uses MMSE equalizers, is written in the form of $\boldsymbol{P}_{m}=\boldsymbol{F} \boldsymbol{\Phi}_{m}$, which is also based on OFDM signalling with per-subcarrier power control. However, the optimal power allocation differs from the one for maximizing capacity; the optimal power allocation is given as follows:

$$
\phi(i)=\frac{\sigma^{2} \Psi_{1}(i)}{\left(\sigma^{2}+\left|\Psi_{1}(i)\right|^{2}\right)^{2}} \boldsymbol{\Theta}(i) \boldsymbol{g}(i),
$$


where $\Psi_{1}(i)$ must fulfil

$$
\left|\Phi_{1}(i)\right|^{2}=\left(\sqrt{\sigma^{2} \xi(i)}-\sigma^{2}\right)_{+0} .
$$

It requires a modification from conventional water filling. Without interference limitation $\left(\lambda_{n}=0\right.$ for all $\left.2 \leq n \leq N\right)$, the $m$-th element of the optimal power in (24) reduces to

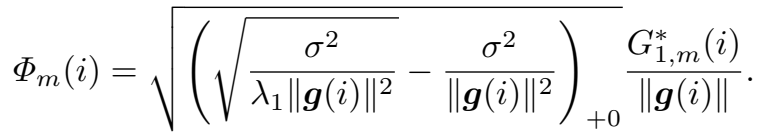

With the optimal precoder, the minimized MSE is written as

$$
\varepsilon=\frac{1}{L} \sum_{i=0}^{L-1}\left(1+\frac{1}{\sigma^{2}}\left|\Psi_{1}(i)\right|^{2}\right)^{-1}=\frac{1}{L} \sum_{i=0}^{L-1}\left(\left(\frac{\xi(i)}{\sigma^{2}}\right)_{+1}\right)^{-\frac{1}{2}}
$$

\section{SC VERSUS TR: CAPACITY SCALING LAW}

This section describes a scaling law which shows the capacity advantage of TR over SC with respect to the number of distributed antennas $M$. We confirm that the TR does not offer better performance than does the SC for the high SNR regime if we use only one transmitter. However, distributed antenna systems enable the TR to outperform the SC. We derive the following scaling law:

- For high SNRs, the capacity gap between TR and SC has asymptotically a logarithmic increase as a function of $M$.

- For low SNRs, the capacity ratio between TR and SC has a linear increase with $M$.

\section{A. Unitary Precoder and TR Precoder}

The SC precoder $\left(\boldsymbol{P}_{m}=\eta_{m} \boldsymbol{I}_{L}\right)$ gives the following capacity:

$$
C_{\mathrm{SC}}=\frac{1}{L} \sum_{i=0}^{L-1} \log \left(1+\frac{1}{\sigma^{2}}\left|\sum_{m} \eta_{m} G_{1, m}(i)\right|^{2}\right) .
$$

When each distributed transmitter has an identical transmission power, we can write $\eta_{m}^{2}=E_{\mathrm{s}}$ for all $1 \leq m \leq M$. Note that any arbitrary unitary precoding (including OFDM without power control, i.e., $\boldsymbol{P}_{m}=\eta_{m} \boldsymbol{F}$ ) offers exactly the same capacity (and MSE) as the one given above. Hence, OFDM without per-subcarrier power control has no advantage over the SC transmission.

In contrast, the TR precoder $\left(\boldsymbol{P}_{m}=\eta_{m} \boldsymbol{H}_{1, m}^{\dagger}\right)$ gives the following capacity:

$$
C_{\mathrm{TR}}=\frac{1}{L} \sum_{i=0}^{L-1} \log \left(1+\frac{1}{\sigma^{2}}\left(\sum_{m} \eta_{m}\left|G_{1, m}(i)\right|^{2}\right)^{2}\right) .
$$

For the case when the transmission power is identical for all the distributed antennas, we have $\eta_{m}^{2}=E_{\mathrm{s}} / \bar{\gamma}_{m}^{2}$ where $\bar{\gamma}_{m}^{2}=$ $\frac{1}{L} \sum_{i}\left|G_{1, m}(i)\right|^{2}$ is the average channel gain.

\section{B. Capacity Scaling Law}

1) High SNR Regime: Assume a high SNR regime such that $E_{\mathrm{s}} / \sigma^{2} \gg 1$, we can approximate

$$
\begin{aligned}
C_{\mathrm{SC}} & \simeq \frac{1}{L} \sum_{i=0}^{L-1} \log \frac{E_{\mathrm{s}}}{\sigma^{2}}\left|\sum_{m} G_{1, m}(i)\right|^{2}, \\
C_{\mathrm{TR}} & \simeq \frac{1}{L} \sum_{i=0}^{L-1} \log \frac{E_{\mathrm{s}}}{\sigma^{2}}\left(\sum_{m} \frac{1}{\bar{\gamma}_{m}}\left|G_{1, m}(i)\right|^{2}\right)^{2},
\end{aligned}
$$

respectively for the $\mathrm{SC}$ and $\mathrm{TR}$ precoders. Assuming that $G_{n, m}(i)$ has a complex Gaussian distribution, the channel coherence bandwidth is much smaller than the considered system bandwidth, and $\bar{\gamma}_{m}=1$ for large packet length, the probability distribution functions of $\left|\sum G_{1, m}(i)\right|^{2}$ and $\sum\left|G_{1, m}(i)\right|^{2}$ can follow the exponential distribution with an average of $M$ and the chi-square distribution with $2 M$ degrees of freedom, respectively. Therefore, we can approximate

$$
\begin{aligned}
C_{\mathrm{TR}}-C_{\mathrm{SC}} & \simeq \int_{0}^{\infty} \log \left(t^{2}\right) \frac{t^{M-1}}{(M-1) !} \mathrm{e}^{-t} \mathrm{~d} t-\int_{0}^{\infty} \log (t) \frac{1}{M} \mathrm{e}^{-\frac{t}{M}} \mathrm{~d} t \\
& =2 \sum_{m=1}^{M-1} \frac{1}{m}-\left(\gamma_{\mathrm{E}}+\log (M)\right),
\end{aligned}
$$

where $\gamma_{\mathrm{E}} \simeq 0.58$ is the Euler's constant. Since the harmonic number $\sum_{m}^{M} 1 / m$ can be well approximated by $\gamma_{\mathrm{E}}+\log (M)$ for large $M$, we can see that $C_{\mathrm{TR}}-C_{\mathrm{SC}} \simeq \gamma_{\mathrm{E}}+\log (M)$ is logarithmically increased with $M$, which implies that a larger number of distributed antennas can improve more the advantage of TR over SC. Note that $C_{\mathrm{SC}}>C_{\mathrm{TR}}$ by $\gamma_{\mathrm{E}}$ for $M=1$, while otherwise $C_{\mathrm{SC}}<C_{\mathrm{TR}}$. Therefore, TR can be worse than SC by $\gamma_{\mathrm{E}} / \log (2) \simeq 0.84 \mathrm{bps} / \mathrm{Hz}$ for high SNR in the single antenna case. The opposite happens for the case in which multiple antennas are used: when $M=2$, the achievable gain is $C_{\mathrm{TR}}-C_{\mathrm{SC}} \simeq 1.05 \mathrm{bps} / \mathrm{Hz}$.

2) Low SNR Regime: For low SNR such that $E_{\mathrm{s}} / \sigma^{2} \ll 1$, approximating $\log (1+t) \simeq t$ for $|t| \ll 1$, we obtain the following

$$
\begin{aligned}
C_{\mathrm{SC}} & \simeq \frac{E_{\mathrm{s}}}{\sigma^{2}} \int_{0}^{\infty} t \frac{1}{M} \mathrm{e}^{-\frac{t}{M}} \mathrm{~d} t=\frac{E_{\mathrm{s}}}{\sigma^{2}} M, \\
C_{\mathrm{TR}} & \simeq \frac{E_{\mathrm{s}}}{\sigma^{2}} \int_{0}^{\infty} t^{2} \frac{t^{M-1}}{(M-1) !} \mathrm{e}^{-t} \mathrm{~d} t=\frac{E_{\mathrm{s}}}{\sigma^{2}} M(M+1) .
\end{aligned}
$$

Therefore, TR offers always better capacity than SC for low $\mathrm{SNR}$; more specifically, the capacity ratio $C_{\mathrm{TR}} / C_{\mathrm{SC}} \simeq 1+M$ can be linearly increased with $M$.

\section{CAPACITY AND MSE COMPARISON}

Table I lists capacity comparisons among the several types of precoding schemes we presented in Section II-B, according to the instantaneous channel realization. The transmission energy $\eta_{m}^{2}$ is constrained by the maximum symbol energy $E_{\mathrm{s}}$ and the allowable interference energy $E_{\mathrm{v}}$ as in (15) and (16).

Fig. 2 shows (log-scaled and linear-scaled) Ergodic capacity of various precoding schemes in the case when there is no interference limitation, i.e. $E_{\mathrm{v}}=\infty$. For these simulations, we assume that the power delay profile consists of $P_{\mathrm{ch}}=16$ 
TABLE I

CAPacity Comparison among Various Types of Linear Block Precoding

\begin{tabular}{lll}
\hline Precoding Scheme & Capacity $C$ & Energy limitation $\eta_{m}^{2}$ \\
\hline Optimum (OFDM) & $\frac{1}{L} \sum \log \left(1+\frac{1}{\sigma^{2}}\left|\Psi_{1}(i)\right|^{2}\right)$ & $\left|\Psi_{1}(i)\right|^{2}$ is given by modified water filling in (22) or (25) \\
Unitary (SC) & $\frac{1}{L} \sum \log \left(1+\frac{1}{\sigma^{2}}\left|\sum \eta_{m} G_{1, m}(i)\right|^{2}\right)$ & $\frac{1}{M} \sum \eta_{m}^{2} \leq E_{\mathrm{s}}, \frac{1}{M L} \sum\left|\sum \eta_{m} G_{n, m}(i)\right|^{2} \leq E_{\mathrm{v}}$ \\
MRC-TR & $\frac{1}{L} \sum \log \left(1+\frac{1}{\sigma^{2}}\left(\sum \eta_{m}\left|G_{1, m}(i)\right|^{2}\right)^{2}\right)$ & $\frac{1}{M L} \sum \eta_{m}^{2} \sum\left|G_{1, m}(i)\right|^{2} \leq E_{\mathrm{s}}, \frac{1}{M L} \sum\left|\sum \eta_{m} G_{n, m}(i) G_{1, m}^{*}(i)\right|^{2} \leq E_{\mathrm{v}}$ \\
EGC-TR, SLC-TR & $\frac{1}{L} \sum \log \left(1+\frac{1}{\sigma^{2}}\left|\sum \eta_{m} G_{1, m}(i) \breve{G}_{1, m}(i)\right|^{2}\right)$ & $\frac{1}{M L} \sum \eta_{m}^{2} \sum\left|\breve{G}_{1, m}(i)\right|^{2} \leq E_{\mathrm{s}}, \frac{1}{M L} \sum\left|\sum \eta_{m} G_{n, m}(i) \breve{G}_{1, m}^{*}(i)\right|^{2} \leq E_{\mathrm{v}}$ \\
ZF & $\log \left(1+\frac{1}{\sigma^{2}}\left(\sum \eta_{m}\right)^{2}\right)$ & $\frac{1}{M L} \sum \eta_{m}^{2} \sum \frac{1}{\left|G_{1, m}(i)\right|^{2}} \leq E_{\mathrm{s}}, \frac{1}{M L} \sum\left|\sum \eta_{m} \frac{G_{n, m}(i) G_{1, m}^{*}(i)}{\left|G_{1, m}(i)\right|^{2}}\right|^{2} \leq E_{\mathrm{v}}$ \\
MMSE & $\frac{1}{L} \sum \log \left(1+\frac{1}{\sigma^{2}}\left(\sum \eta_{m} \frac{\left|G_{1, m}(i)\right|^{2}}{\left|G_{1, m}(i)\right|^{2}+\sigma^{2}}\right)^{2}\right)$ & $\frac{1}{M L} \sum \eta_{m}^{2} \sum \frac{\left|G_{1, m}(i)\right|^{2}}{\left(\left|G_{1, m}(i)\right|^{2}+\sigma^{2}\right)^{2}} \leq E_{\mathrm{s}}, \frac{1}{M L} \sum\left|\sum \eta_{m} \frac{G_{n, m}(i) G_{1, m}^{*}(i)}{\left|G_{1, m}(i)\right|^{2}+\sigma^{2}}\right|^{2} \leq E_{\mathrm{v}}$ \\
\hline
\end{tabular}

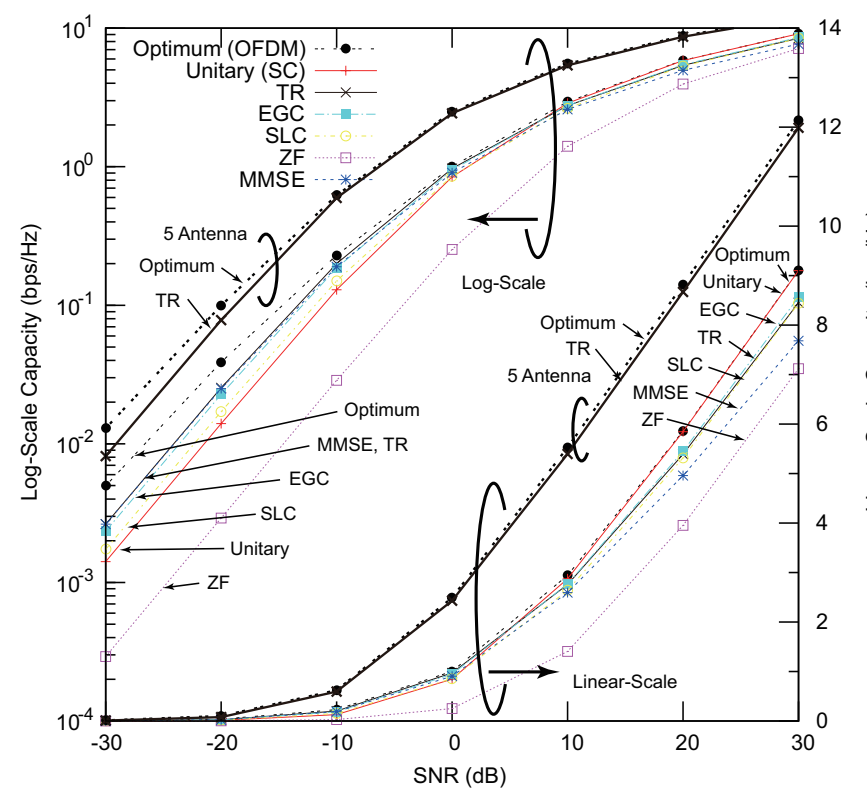

Fig. 2. Achievable capacity as a function of SNR in $1.0 \mathrm{~dB}$ decaying 16-path Rayleigh fading $(M=1$ and $M=5)$.

sample-spaced paths whose power is exponentially decreasing with increasing delay by $1 \mathrm{~dB}$ each. We set $L=512$. From Fig. 2, one can see that the TR schemes are better than preequalization schemes, whereas those are worse than SC by approximately $0.8 \mathrm{bps} / \mathrm{Hz}$ for a high SNR as we discussed in the previous section. The OFDM with optimal power allocation has no visible advantage over SC. However, OFDM outperforms SC for a low SNR. In a low SNR, TR can offer twice as large a capacity as SC. It can be observed that the use of multiple antennas $(M=5)$ can significantly improve the capacity.

In Fig. 3, we show the impact of the number of distributed antennas $M$ on the achievable capacity for an SNR of $\pm 30 \mathrm{~dB}$. We can see that the unitary precoder (SC and OFDM without power control) cannot enjoy any benefit even if we increase the number of distributed antennas. For low SNR, TR offers the capacity comparable to the one achieved by MMSE, and TR is always better than the unitary precoding. Note that the simulation result of capacity ratio $C_{\mathrm{TR}} / C_{\mathrm{SC}}$ completely matches the scaling law of $1+M$. For high SNR, TR is worse than $\mathrm{SC}$ by $0.8 \mathrm{bps} / \mathrm{Hz}$ when $M=1$, whereas it outperforms

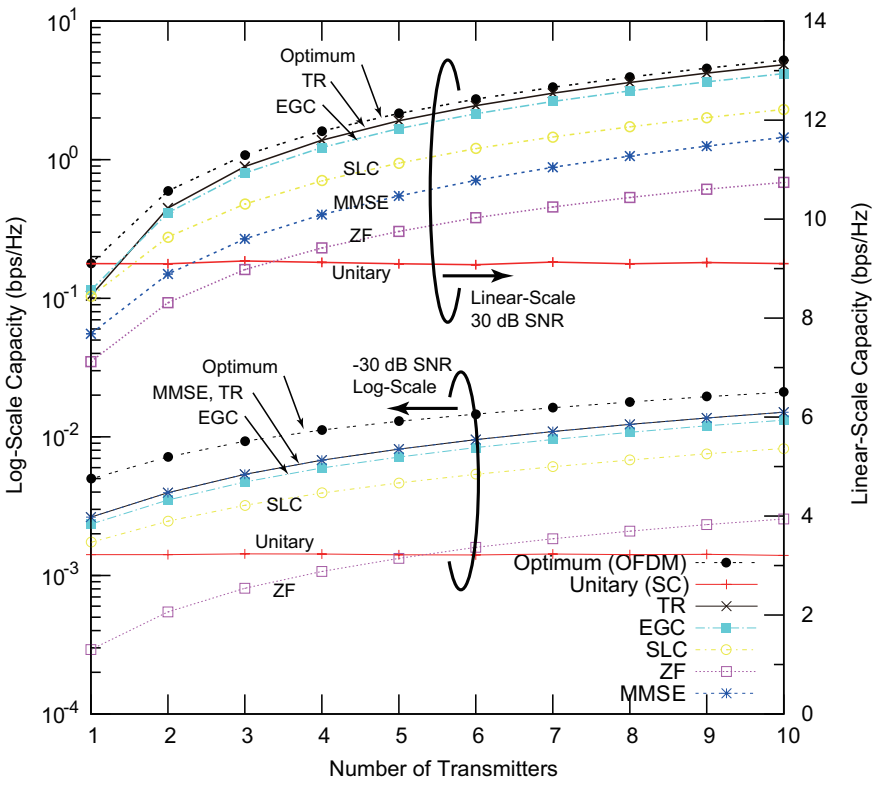

Fig. 3. Achievable capacity versus the number of distributed antennas in $1.0 \mathrm{~dB}$ decaying 16-path Rayleigh fading ( $\pm 30 \mathrm{~dB}$ SNR).

SC with multiple transmitters. Moreover, the TR can approach the optimal precoder (OFDM with optimal power control) for large $M$. The capacity advantage of $C_{\mathrm{TR}}-C_{\mathrm{SC}}$ perfectly agrees with the scaling law of $\sum^{M-1} 1 / m-\left(\gamma_{\mathrm{E}}+\log (M)\right)$.

In Fig. 4, we plot the MSE curves with no interference limitation as a function of the number of transmitters $M$ at an SNR of $0 \mathrm{~dB}$ and $30 \mathrm{~dB}$. At high SNR, the original MRCTR scheme has the worst performance in MSE among these precoding schemes for $M=1$, whereas it can outperform all the other non-optimal precoders when $M \geq 3$. The MSE of TR can converge to that of the optimal precoder.

In Fig. 5, we evaluate MSE versus the number of receivers $N$ for an interference-to-noise ratio (INR) of $M E_{\mathrm{v}} / \sigma^{2}=$ $20 \mathrm{~dB}$ and an SNR of $M E_{\mathrm{s}} / \sigma^{2}=30 \mathrm{~dB}$. Due to the interference limitation, the MSE can be degraded when $N>1$. However, the MSE degradation can be saturated for morethan four antennas. In this figure, we also present the MSE curves when the cyclic prefix length is not sufficient to avoid inter-symbol interference, i.e., $L_{\mathrm{cp}}<15$. We can see that the decrease of the cyclic prefix length can degrade MSE especially for the unitary (SC) precoder. Note that the TR 


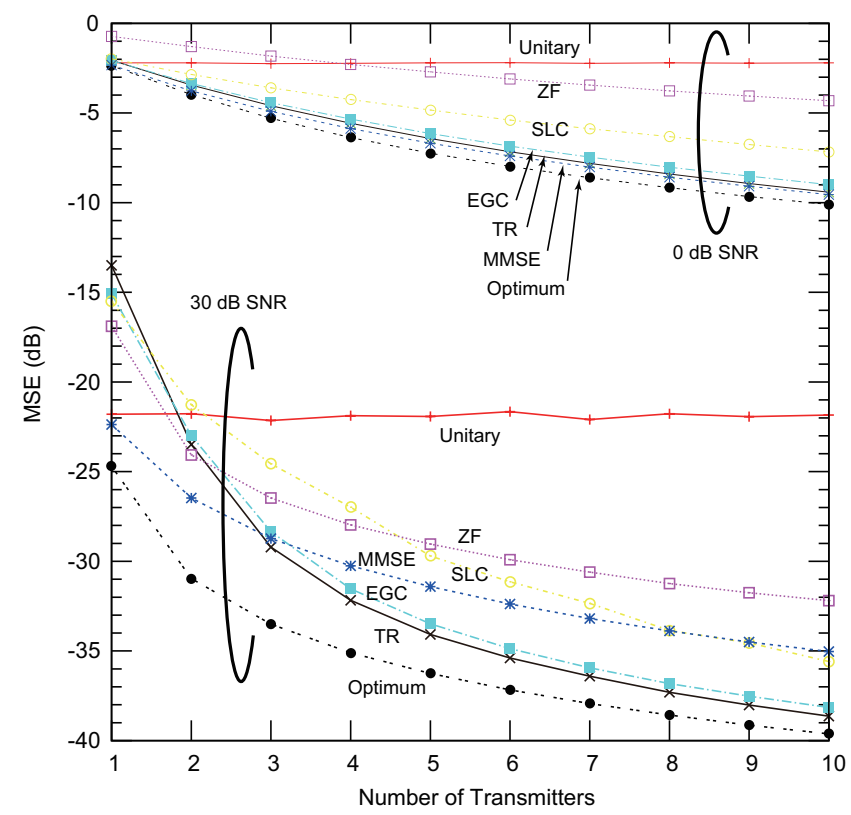

Fig. 4. MSE versus the number of transmitters in $1.0 \mathrm{~dB}$ decaying 16-path Rayleigh fading $(0 \mathrm{~dB}$ and $30 \mathrm{~dB} \mathrm{SNR})$.

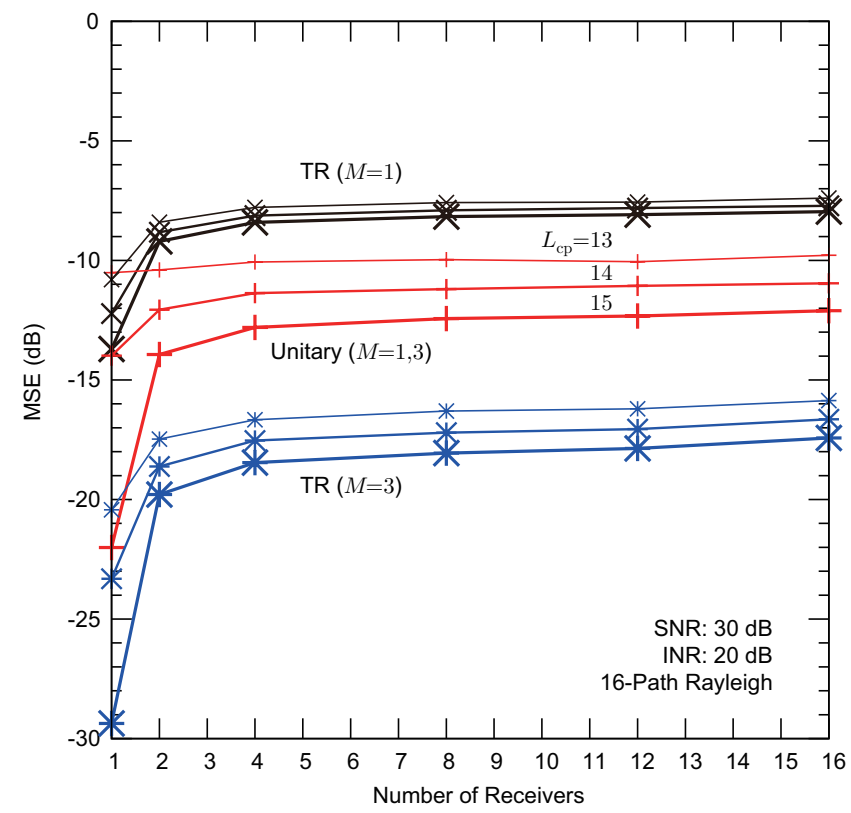

Fig. 5. MSE versus the number of receivers in $1.0 \mathrm{~dB}$ decaying 16-path Rayleigh fading ( $30 \mathrm{~dB}$ SNR, $20 \mathrm{~dB}$ INR, $M \in\{1,3\}, L_{\mathrm{cp}} \in\{13,14,15\}$ ).

is more robust than the $\mathrm{SC}$ for insufficient $\mathrm{CP}$ lengths because the TR can shorten the channel delay spread.

\section{SUMMARY}

In this paper, we investigated an achievable capacity and MSE for several linear block precoding schemes. We derived optimal power allocation for OFDM in the presence of interference limitation and multiple distributed antennas. We confirmed that the OFDM with optimal power control is advantageous over the SC block transmission in capacity only in the low SNR regime, and in MSE for a high SNR. For single antenna systems, the TR precoding has a capacity loss of approximately $0.8 \mathrm{bps} / \mathrm{Hz}$ over SC for a high SNR, while it offers double the capacity of SC for a low SNR. We derived a capacity scaling law which shows how the number of transmitters can increase the capacity: The capacity difference between TR and SC has a logarithmic increase with the number of transmitters for high SNR, and the capacity ratio has a linear increase for low SNR. In distributed antenna systems, the TR gives a high capacity which is close to the one achieved by the optimal precoder.

Capacity and MSE are, of course, not the only relevant parameters for the selection of a transmission scheme. For example, SC generally provides lower peak-to-average power ratio for transmissions, making it easier to build suitable power amplifiers; TR provides channel shortening which can decrease the required length of the $\mathrm{CP}$, and also allows to shift complexity from the receiver to the transmitter, which is often desirable. Nevertheless, our unified evaluation methodology still allows us to gain new insights into the most fundamental characteristics of a very important class of high-speed digital transmission schemes.

\section{REFERENCES}

[1] A. F. Molisch, Wireless Communications, John Wiley \& Sons, 2005.

[2] P. Kyritsi, G. Papanicolaou, P. Eggers, and A. Oprea, "Time reversal techniques for wireless communications" 2004.

[3] J. M. F. Moura and Y. Jin, "Detection by time reversal: single antenna," IEEE Trans. Signal Proces., vol. 51, no. 1, pp. 187-201, Jan. 2007.

[4] D. D. Stancil, A. G. Cepni, B. E. Henty, Y. Jiang, Y. Jin, J.-G. Zhu, and J. M. F. Moura, "Super-resolution focusing and nulling in rich multipath environments using time-reversal techniques," IEEE ICEAA, Apr. 2005.

[5] G. Ding, D. Wang, and Z. Chen, "Performance evaluation of two kinds of precoding joint with MMSE equalization in DS-UWB," ChinaCom, pp. 1-5, Oct. 2006.

[6] P. Kyritsi, G. Papanicolaou, P. Eggers, and A. Oprea, "MISO time reversal and delay-spread compression for FWA channels at $5 \mathrm{GHz}$," IEEE Antennas. Wireless Propag. Lett., vol. 3, pp. 96-99, 2004.

[7] H. T. Nguyen, "On the performance of one bit time reversal for multiuser wireless communciations," IEEE ISWCS, pp. 672-676, 2007.

[8] H. T. Nguyen, P. Kyritsi, and P. C. F. Eggers, "Time reversal technique for multi-user wireless communication with single tap receiver,"

[9] P. Kyritsi, P. Stoica, G. Papanicolaou, P. Eggers, and A. Oprea, "Time reversal and zero-forcing equalization for fixed wireless access channels," 2005.

[10] H. T. Nguyen, J. B. Andersen, G. F. Pedersen, P. Kyritsi, and P. C. F Eggers, "Time reversal in wireless communications: A measurementbased investigation," IEEE Trans. Wireless Commun., vol. 5, no. 8, pp. 2242-2252, Aug. 2006

[11] H. T. Nguyen, J. B. Andersen, and G. F. Pedersen, "The potential use of time reversal techniques in multiple element antenna systems," IEEE Commun. Lett., vol. 9, no. 1, pp. 40-42, Jan. 2005.

[12] Y. Jin, Y. Jiang, and J. M. F. Moura, "Multiple antenna time reversal transmission in ultra-wideband communications," IEEE GLOBECOM, Nov. 2007.

[13] R. C. Qiu, C. J. Zhou, N. Guo, and J. Q. Zhang, "Time reversal with MISO for ultrawideband communications: Experimental results," IEEE Trans. Wireless Propag. Lett., vol. 5, pp. 269-273, 2006.

[14] C. Zhou, N. Guo, and R. C. Qiu, "Experimental results on multipleinput single-output (MISO) time reversal for UWB systems in an office environment," IEEE MILCOM, 2007.

[15] H. T. Nguyen, I. Z. Kovács, and P. C. F. Eggers, "A time reversal transmission approach for multiuser UWB communications," IEEE Trans. Antennas Propag., vol. 54, no. 11, pp. 3216-3224, Nov. 2006. 